\title{
Comment on Octet Baryon Magnetic Moments in the Chiral Quark Model with Configuration Mixing
}

\author{
Jerrold Franklin* \\ Department of Physics \\ Temple University Philadelphia, PA 19122-6082
}

(July 17, 1998)

\begin{abstract}
The importance of exchange currents, and of conserving isotopic spin at both the quark and baryon levels in application of the chiral quark model to any calculation of baryon magnetic moments is emphasized.
\end{abstract}

*Internet address: V5030E@VM.TEMPLE.EDU 
The original static quark model made predictions for baryon magnetic moments [1] that were in remarkable qualitative agreement with early magnetic moment measurements. However, more accurate measurements of the magnetic moments of the baryon octet differ from the static quark model predictions by up to 0.2 nuclear magnetons. This difference has generally been attributed to various "non-static" effects in the quark model. Some time ago, the sum rule [2]

$m u(p)-m u(n)+m u\left(\operatorname{Sigma}^{-}\right)-m u\left(\operatorname{Sigma}^{+}\right)+m u\left(X i^{0}\right)-m u\left(X i^{-}\right)=0 q u a d(0.49 p m .05)$,

was derived, with the prediction that this linear combination of octet baryon moments should vanish, even in the presence of a general class of non-static effects. For this combination of baryons, the non-static magnetic moment contributions would cancel out if the ultimate contribution from each quark were independent of which baryon the quark was in. This "baryon independence" would follow, for instance, if the non-static parts of the baryon wave functions were $\mathrm{SU}(3)$ symmetric. Because of the cancellation of the non-static contributions, it was expected that the sum rule of Eq. (回) would be in better agreement with experiment than individual quark moments. However, subsequent tests of the sum rule [3] showed that it disagreed with experiment by more than did any single magnetic moment. The most recent experimental value [4] for the sum rule is shown in parentheses in Eq. (11). This violation of the sum rule indicates strong $\mathrm{SU}(3)$ breaking and baryon dependent non-static contributions to the baryon magnetic moments.

A recent paper "Octet Baryon Magnetic Moments in the Chiral Quark Model with Configuration Mixing" [5] has presented the conclusion that the sum-rule of Eq. (11) is not broken by arbitrary SU(3) symmetry breaking in the chiral quark model. However, the application of the chiral quark model in Ref.citelos leaves out important exchange effects that are as large as the effects considered in Ref. citelos. These exchange effects must enter in any model if conservation of isotopic spin is imposed at both the quark and the baryon level. Proper inclusion of exchange effects would produce a non zero contribution to the sum rule of Eq. (11). The conclusion in Ref. [0] is also contradicted by an explicit calculation [6] of $\mathrm{SU}(3)$ symmetry breaking within a class of models that includes the chiral quark model.

An important omission in Ref.citelos is the lack of conservation of isotopic spin in its effective baryon wave functions, and the consequent lack of exchange currents. The assumption is made in Ref. citelos that the interaction of the GBs (Goldstone bosons) is weak enough to be treated by perturbation theory. This assumption is used to write down effective quark wave functions in first order perturbation theory in Eqs. (2) of Ref. [5]. However, because of the degeneracy of the baryons within each isomultiplet, degenerate perturbation theory must be used. The appropriate I-spin linear combinations of both baryon-GB and quark-GB must be used in the perturbation expansion of the baryon wave functions. Imposing isotopic spin at the baryon level requires the inclusion of pion exchange currents, which are absent in Ref. [5].

Exchange currents would show up as the important process of a Goldstone boson being emitted by one quark, and reabsorbed by a different quark in the same baryon. This emission and reabsorption would be equivalent to exchange currents, which should contribute to the baryon magnetic moments. Without this process, the Goldstone boson emission considered 
in Ref. [5] can only affect the effective anomalous magnetic moments of the quarks. Because the reabsorption of the Goldstone boson by a different quark is left out, the quark "transition probabilities" listed in Eqs. (A3-A5) of the appendix of Ref. [5] do not depend on which baryon the quark is in. The transition probabilities in Ref. [5] are baryon independent in the sense of Ref. [2], and therefor the magnetic moments calculated in Ref. [5] satisfy the sum rule of Eq. (11).

With the inclusion of $\mathrm{SU}(3)$ breaking pion exchange currents as described above, the quark transition probability for the $u$ quark in a proton would be different than that for a $\mathrm{u}$ quark in a $\Sigma^{+}$. That is because a $\mathrm{u}$ quark in a proton can emit a $\pi^{+}$that is reabsorbed by a d quark in same proton. There is no mechanism for this to happen in a $\Sigma^{+}$hyperon where there is no d quark to reabsorb the $\pi^{+}$. Similarly, there would be a difference in the quark transition probabilities between the $d$ quark in the proton and the d quark in the $\Xi^{-}$. With SU(3) breaking (in the form of pion dominance), the quark transition probabilities would not be baryon independent, and the resulting baryon magnetic moments would break the sum rule of Eq. (11). Independently of how the quarks emit or reabsorb Goldstone bosons, pion exchange currents are required if isotopic spin is conserved at both the quark and baryon levels [6]. This means that baryon dependent quark contributions to baryon magnetic moments are required in any theory with full conservation of isotopic spin, in the absence of $\mathrm{SU}(3)$ symmetry. If $\mathrm{SU}(3)$ symmetry were preserved in the GB emission, then kaon and $\eta$ exchange currents would compensate for the pion exchange currents, preserving the sum rule.

An explicit example where the sum rule of Eq. (1) does not hold in a model that breaks $\mathrm{SU}(3)$ symmetry is given in Ref. [6]. There, the pions, because of their anomalously light mass, are taken to dominate the meson exchange currents, while $\mathrm{k}$ meson currents are left out. The pion exchange currents break $\mathrm{SU}(3)$, and the resulting baryon moments do not satisfy the sum rule of Eq. (1). For the quark model with pion contributions (including exchange), the prediction from the "QM+pion" column of Table I of Ref. [6] for the sum rule of Eq. (1) is 0.39, which is close to the experimental value.

The model in Ref.citejfpi is quite general in that the baryon wave functions do not depend on a specific boson emission mechanism. The wave functions are constrained only by conservation of isotopic spin, angular momentum, and parity on both the quark and on the baryon levels. Any model, including chiral perturbation theory, that satisfied these symmetries at both levels would be consistent with Ref. [6]. In particular, chiral perturbation theory (with $\mathrm{k}$ and $\eta$ mesons left out) should agree with the eight equations (A1-A8) for baryon magnetic moments listed in the Appendix of Ref. [6]. Those eight baryon moments are given in terms of five parameters (bare $d$ quark moment, bare s quark moment, pion probability in the nucleon, effective pion orbital magnetic moment, relative importance of decuplet baryons in the octet baryon wave function). As long as the pion probability is non-zero, the baryon moments given by the eight equations will violate the sum rule in Eq. (1). A more detailed theory could predict values for the parameters, but could not change the parametrization without violating isotopic spin or angular momentum conservation.

The authors of Ref.citelos do conclude that configuration mixing of SU(3) symmetric gluons [7] or $\mathrm{SU}(3)$ symmetric diquarks [8] can produce a non zero result for the sum rule of Eq. (1). This is surprising, because $\mathrm{SU}(3)$ symmetric mechanisms should not affect the sum rule. The reason that these mechanisms do contribute to the sum rule in Ref. [5] is 
that different mixing angles are chosen for different baryons in these cases, which breaks the SU(3) symmetry. The contribution of Goldstone boson emission can also be treated in terms of configuration mixing of the GBs in the same way as Ref. [5] does for gluons and diquarks. In fact, this is the method used in Ref. [6]. In the case of an SU(3) breaking GB admixture, the mixing angles are required to be different for baryons of different strangeness because of the pion exchange mechanism discussed above. This is another way to see that breaking $\mathrm{SU}(3)$ symmetry in the emission of Goldstone bosons must break the sum rule of Eq. (1). 


\section{REFERENCES}

[1] G. Morpurgo, Phys. 2, 95 (1965); W. Thirring, Acta Phys. Austriaca Supp. II, 205 (1965); H. R. Rubinstein, F. Scheck, and R. Socolow, Phys. Rev. 154, 1608 (1967); J. Franklin, ibid. 172, 1807 (1968).

[2] J. Franklin, Phys. Rev. 182, 1607 (1969).

[3] J. Franklin, Phys. Rev. D 20, 1742 (1979); ibid. 29, 2648 (1984); G. Karl, ibid. 45, 247 (1992).

[4] Particle Data Group, R. M. Barnett et al. ,Phys. Rev. D 54, 1 (1996).

[5] J. Linde T. Ohlsson, and H. Snellman, Phys. Rev. D 57, 452 (1998)

[6] J. Franklin, Phys. Rev. D 30, 1542 (1984).

[7] H. J. Lipkin, Phys. Lett. B 251, 613 (1990).

[8] J. Carroll, D. B. Lichtenberg, J. Franklin, Phys. Rev. 174, 1681 (1968). 